\title{
Reply to "Comment on 'Investigating hydrogen dependence of dynamics of confined water: Monolayer, hydration water and Maxwell-Wagner processes' [J. Chem. Phys. 133, 037101 (2010)]"'
}

\author{
Johan Sjöström, ${ }^{1, a)}$ Jan Swenson, ${ }^{1}$ Rikard Bergman, ${ }^{1}$ and Shigeharu Kittaka ${ }^{2}$ \\ ${ }^{1}$ Department of Applied Physics, Chalmers University of Technology, SE-41296 Göteborg, Sweden \\ ${ }^{2}$ Department of Chemistry, Faculty of Science, Okayama University of Science, \\ 1-1 Ridaicho, Okayama 700-0005, Japan
}

(Received 2 November 2009; accepted 23 February 2010; published online 19 July 2010)

[doi:10.1063/1.3451103]

The preceding comment by Ryabov et al. ${ }^{1}$ raises several questions regarding the conclusions of our recent paper $^{2}$ concerning a highly anomalous process observed in dielectric studies of water in hard confinements. We show that the model of interfacial polarization used in Ref. 2 can describe the observed phenomena and that Ryabov et al. unfortunately misinterpreted the model. Our responses to the comments are as follows.

Raw data in the complete frequency range are not shown since the low and high frequency ranges are usually not measured on the same batches. One example of the full spectrum is, however, shown in Ref. 3, and we show relaxation times for the whole frequency rage. The range of the nonmonotonic process never reaches above $100 \mathrm{kHz}$.

We note that the tick mark labels of the frequency axes in Fig. 2 of Ref. 2 are unfortunately permuted. All processes enter the spectrum at low frequencies when increasing the temperature and not the other way around.

Some of the water in the larger pores (pore diameter of $3.6 \mathrm{~nm}$ ) is crystalline at low temperatures. The ice melts at $240 \mathrm{~K}$. This changes the spectrum, especially at higher frequencies. We have not included this in the discussion more than noted the change in the relaxation behavior at this temperature for the nonmonotonic process as the level of liquid water increases.

The used hydration process is by no means a guarantee for complete filling of the pores. However, detailed studies of pore filling by thermal gravimetric analysis (Ref. 4) show that we almost reach complete filling by this hydration process, as assumed in the model for simplicity. Still, the absolute amount of water in the pores does not change the qualitative features of the results.

The nature of the low temperature process in the spectrum is a very debated subject itself and many different denotations can be found in the literature. This process is shown to be a general and local feature in most aqueous systems and carries many features that are icelike, as stated by Ryabov et al., even though present in completely amorphous systems. ${ }^{5,6}$

The Teflon film changes the spectrum as an additional capacitance is added. However, the nonmonotonic process is clearly visible both with and without the film. Without the film the process is sometimes hidden by a low frequency dispersion that, as pointed out by Ryabov et al., may be caused by outer surface water. We can conclude that the qualitative features of the process (nonmonotonicity, temperature-, and frequency ranges) are preserved as we introduce the film.

Let us now focus on the main issue of the comment; the model of a Maxwell-Wagner (MW) process resulting from spherical heterogeneities in an insulating material. Unfortunately, some subscripts were missing in Eq. (6) of Ref. 2 and perhaps the model is insufficiently explained such that it is misinterpreted by Ryabov et al. The assumptions of the model are clarified here and we use different, hopefully more suitable subscripts. The model is a two-component model of spherical voids in a silica matrix. Since the voids are initially filled with water the permittivity and conductivity within the voids is that of milliQ-water (conductivity $<0.1 \mu \mathrm{S} / \mathrm{m}$ ) at low temperatures. As water content is decreased on increasing the temperature, as shown in Refs. 2 and 7, we change the permittivity and conductivity of the voids in a linear manner with respect to the amount of water still residing in the voids. In the model ${ }^{2}$ the loss of water, $y(T)$, is given in a linear and a quadratic form with respect to temperature. The volume of the voids is constant throughout the temperature range and the relation $v_{\text {matrix }}+v_{\text {void }}=1$ holds. Thus, the permittivity $\varepsilon^{\prime}$ and conductivity $\sigma$ of the voids changes by two factors: (i) the change of permittivity and conductivity of water with temperature and (ii) a linear relationship with the water content in the voids: $\sigma_{\text {voids }}=\sigma_{\text {water }}(T) y(T), \varepsilon^{\prime}{ }_{\text {void }}(T)$ $=\varepsilon^{\prime}{ }_{\text {water }}(T) y(T)$. In Ref. 2 the subscripts void and matrix are labeled $w$ and $s$, respectively. The characteristic relaxation time of the additional process in the spectrum is then

$$
\tau_{\mathrm{MW}}=\varepsilon_{0} \frac{2 \varepsilon^{\prime}{ }_{\text {matrix }}+\varepsilon^{\prime}{ }_{\text {void }}-v_{\text {void }}\left(\varepsilon^{\prime}{ }_{\text {void }}-\varepsilon^{\prime}{ }_{\text {matrix }}\right)}{v_{\text {matrix }} \sigma_{\text {void }}}
$$

where $\varepsilon_{0}$ is the vacuum permittivity and $v$ is the fractional volume of voids and the matrix. Using these assumptions, $\tau_{\mathrm{MW}}$ exhibits a nonmonotonic temperature dependence as shown in Fig. 1. The qualitative feature of the relaxation time reflects that observed in experiments. ${ }^{2,7}$ The reason why this is strikingly similar to the relaxation map shown in Ref. 1 is that the authors misinterpreted the model such that the volume of the voids changed with temperature as $y(T)$. Thereafter, by exchanging the volumes of matrix and void in Eq. (1), one recaptures the $1 / y(T)$ dependence of Eq. (1). Still, as already mentioned, there is no justification for such permutation. 


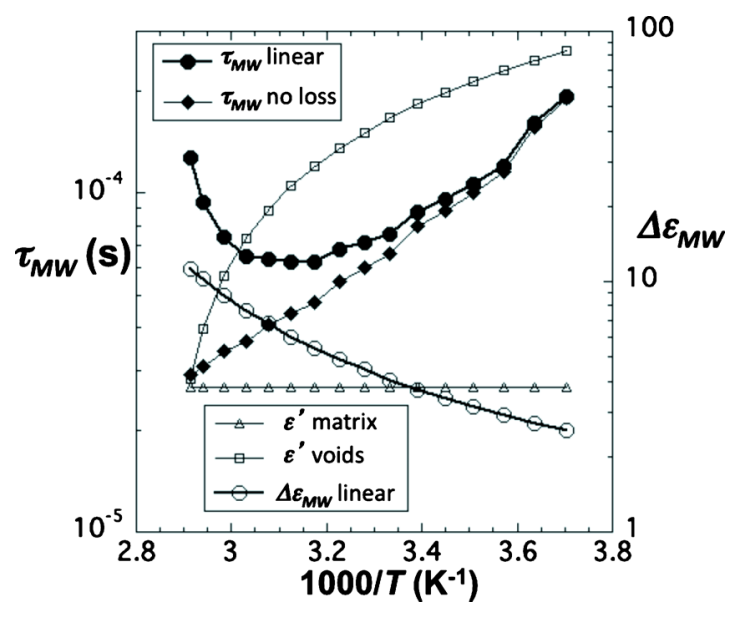

FIG. 1. Results of the model of a MW process emerging from a silica matrix with water filled spherical heterogeneities which loose water in a linear rate with respect to temperature. Symbols show the characteristic relaxation time of the MW process $(\mathbf{)})$, the same relaxation time without any loss of water $(\checkmark)$, dielectric strength of the MW process with loss of water $(\bigcirc)$, permittivity of the voids $(\square)$, and permittivity of the matrix $(\triangle)$.

It is true that the MW process is Debye-like. The process observed in hydrated MCM-41 (Ref. 2) without a Teflon film is well characterized by a very narrow dispersion (Cole-Cole function with $\alpha=0.82$ ). The process found in Refs. 7-10 all exhibited wider dispersions. It should be mentioned that the Debye relaxation behavior should be broadened by a distribution of pore properties, e.g., pore size. MCM-41 has one of the most narrow distributions of pore sizes found in porous silica, and we expect a wider distribution in other systems and thus a wider MW dispersion.

In Fig. 1 it is also evident that the MW process from the model exhibits a monotonically increasing dielectric strength $\Delta \varepsilon$, with values comparable to the ones reported in the experiments. The strength of the process increases even though the permittivity within the voids decreases and the permittivity of the matrix is constant. The process grows stronger even though less water molecules reside in the voids because it is not relaxing water molecules that are the origin of the process presented in this model, rather it is due to the charge accumulation at the borders between regions of different dielectrical heterogeneities.

We hope that we have clarified the framework of the model presented above and shown that it indeed can reproduce a nonmonotonic relaxation time of a MW process in a porous system that looses water. One might raise questions to the validity of the model concerning the linearity between water content and $\varepsilon^{\prime}{ }_{\text {void }}, \sigma_{\text {void }}$, or that the geometry of the model differs from that of experiments. It can be argued that the number of charge carriers at the pore surfaces is constant, thus maintaining a constant conductivity as water evaporates. We base our assumption on experimental results showing that the conductivity of porous silica decreases as hydration level decreases. ${ }^{11}$ In this context it is physically relevant to decrease $\varepsilon^{\prime}{ }_{\text {void }}$ and $\sigma_{\text {void }}$ with decreasing water content. The exact manner in which this occurs is not known to us. We use a linear relation as a first approximation. It is, however, not our intention to simulate the exact results of the experiments. Instead we use a framework as simple as possible to capture the general aspects of heterogeneities and loss of water and show that these features can give rise to the observed phenomena.

We want to clarify that our results do not prove that the explanation given in Ref. 8 is wrong. In that model the slowing down at high temperatures is caused by a decreased defect density in the structure, such that the surface water is glasslike well above room temperature. We just want to point out that other explanations to the admittedly fascinating phenomenon of Refs. 2 and 7-10 are possible. ${ }^{12}$ For instance, the nonmonotonic process could be due to charge accumulation in other heterogeneities than the porous structure of the material. A two-layer system can be formed by a small airgap between the electrodes, and the powder (in the case of MCM-41) and pellets (for other systems). The effect of such an air-gap would disappear with percolating conductivity between the electrodes, which might explain the sudden disappearance of the nonmonotonic process in Fig. 2 of Ref. 2. It could also explain why the process is always visible in the presence of the Teflon film, which thus acts as a more efficient air gap.

In conclusion, there are many possible explanations for this fascinating phenomenon, including those in Refs. 8 and 2. In this context we would like to emphasize the results presented in Fig. 8 of Ref. 2. Here the sample was first cooled with a controlled cooling rate and thereafter measured on heating. After measurements at several higher temperatures the sample is remeasured at $270 \mathrm{~K}$. We find that the relaxation time at $270 \mathrm{~K}$ is slower after excursions to higher temperatures compared to the first time measured. In our opinion this is a strong indication that loss of water causes the slowing down of the process since very few other parameters change during this experimental procedure.

\footnotetext{
${ }^{a)}$ Electronic mail: johan.sjostrom@ chalmers.se.

${ }^{1}$ Y. Ryabov, A. Gutina, Y. Feldman, S. Frunza, L. Frunza, and A. Schönhals, J. Chem. Phys. 133, 037101 (2010).

${ }^{2}$ J. Sjöström, J. Swenson, R. Bergman, and S. Kittaka, J. Chem. Phys. 128, 154503 (2008).

${ }^{3}$ J. Hedström, J. Swenson, R. Bergman, and S. Kittaka, Eur. Phys. J. Spec. Top. 141, 53 (2007).

${ }^{4}$ S. Kittaka, S. Ishimaru, M. Kuranishi, T. Matsuda, and T. Yamaguchi, Phys. Chem. Chem. Phys. 8, 3223 (2006).

${ }^{5}$ S. Cerveny, G. A. Schwartz, R. Bergman, and J. Swenson, Phys. Rev. Lett. 93, 245702 (2004).

${ }^{6}$ J. Sjöström, J. Mattsson, R. Bergman, E. Johansson, K. Josefsson, D. Svantesson, and J. Swenson, "Dielectric secondary relaxation of water in aqueous binary glass-formers" Phys. Chem. Chem. Phys. (in press).

${ }^{7}$ L. Frunza, H. Kosslick, I. Pitsch, S. Frunza, and A. Schönhals, J. Phys. Chem. B 109, 9154 (2005).

${ }^{8}$ Y. Ryabov, A. Gutina, V. Arkhipov, and Y. Feldman, J. Phys. Chem. B 105, 1845 (2001).

${ }^{9}$ A. Gutina, E. Axelrod, A. Puzenko, E. Rysiakiewicz-Pasek, N. Kozlovich, and Y. Feldman, J. Non-Cryst. Solids 302, 235 (1998).

${ }^{10}$ L. Frunza, H. Kosslick, S. Frunza, and A. Schönhals, J. Phys. Chem. B 106, 9191 (2002).

${ }^{11}$ M. Nogami, R. Nagao, and C. Wong, J. Phys. Chem. B 102, 5772 (1998).

${ }^{12}$ Regarding the nonmonotonic behavior in hydrated faujasite molecular sieves. As we have never studied this system we cannot comment on where and how water resides within the structure. From Ref. 10, a decrease of the weight is noticed in the experimental temperature range. A change in water content will change the permittivity and conductivity within different regions of the material and thus change the properties of a possible MW process.
} 